\title{
Using cerebrospinal fluid for the diagnosis of tuberculous meningitis with GeneXpert
}

\author{
To the Editor:
}

We read with interest the systematic review by C. Denkinger and co-workers on the use of the Xpert MTB/ RIF assay for diagnosing extrapulmonary tuberculosis (TB) [1], particularly in light of World Health Organization (WHO) endorsement of Xpert MTB/RIF and its recent policy statement on extrapulmonary TB [1-3]. Given the severe clinical course and high mortality associated with TB meningitis, combined with the lack of TB culture capacity in much of the developing world, WHO has endorsed testing cerebrospinal fluid (CSF) with the automated Xpert MTB/RIF assay as a first-line test over conventional microscopy in patients with suspected TB meningitis [2]. This policy recommendation came as a "strong recommendation given the urgency of rapid diagnosis" despite the "very low quality of evidence" [2].

Indeed, as C. Denkinger and co-workers' review clearly illustrates, the small number of published studies that have compared Xpert testing of CSF with culture use a variety of different testing protocols, differing in the amount of CSF tested and whether the CSF was centrifuged, decontaminated and/or diluted with sample reagent [1]. One of the largest studies performed to date found a significant improvement in the sensitivity of the Xpert MTB/RIF test in CSF when the CSF was centrifuged [4]. However, this was only with unpaired samples from two different groups of patients; when 12 positive paired samples from the same patients were tested, no significant difference was found [4].

Given the variability in sample processing protocols, the WHO expert group highlighted "the need to develop standardised protocols for processing samples for use in subsequent studies employing Xpert MTB/RIF in non-respiratory specimens. The Expert Group recommended that processing protocols not only focus on one sample type in particular but assess differences in performance with modified processing in the individual steps: homogenization, concentration, decontamination, and sample reagent to sample ratio" [5].

In our laboratory, we tested nine different protocols for using CSF in the Xpert MTB/RIF assay, based on the procedures from published studies [1]. Pooled CSF samples were spiked with an attenuated laboratory strain of Mycobacterium tuberculosis (H37Ra), in concentrations ranging from $10^{2}$ to $10^{6} \mathrm{CFU} \cdot \mathrm{mL}^{-1} \cdot 10$ samples were each tested in nine different ways, varying the amount of sample used, if the sample was centrifuged and how much sample reagent was used. Decontamination of samples was not performed, as CSF should otherwise be sterile. The difference in PCR cycle threshold values was compared to evaluate assay performance. As seen in table 1, depending on the protocol used, the difference in cycle threshold values ranged between 0.1 and 1.9 cycles. These differences were similar regardless of initial bacterial

TABLE 1 Comparison of cerebrospinal fluid (CSF) protocols for Xpert MTB/RIF testing

Protocol

\section{Direct testing of CSF}

$0.5 \mathrm{~mL}$

$1.0 \mathrm{~mL}$

$1.5 \mathrm{~mL}$

$2.0 \mathrm{~mL}$

Sample reagent:sample ratio

$1: 1$

$2: 1$

3:1

CSF centrifuged

$4 \mathrm{~mL}$

$8 \mathrm{~mL}$

Replicates of referent sample
Mean PCR cycle

1.9
1.4
Referent
1.5

1.8
0.1
0.2

0.6
0.2
1.7

Data are presented as the threshold difference. ${ }^{\#}$ : direct testing of $1.5 \mathrm{~mL} \mathrm{CSF}$. 
concentration. However, four replicate samples using the same protocol also demonstrated a 1.7 cycle mean difference, similar to the up to 1.9 cycle difference seen across protocols. The similarity in cycle differences suggests that differences in cycle threshold values may be due to intra-assay variation and not related to the sample processing protocol used. These results show that centrifugation may not be necessary for testing CSF. Additionally, as CSF samples tend to be paucibacillary compared to sputum, less sample reagent may be needed to produce an adequate tuberculocidal effect. Further research, ideally on paired samples from TB meningitis patients, will help elucidate the most sensitive and safest method to test CSF with the Xpert MTB/RIF test, particularly in settings that may not have access to centrifugation.

0 @ERSpublications

Cerebrospinal fluid testing with Xpert MTB/RIF may not require complex sample processing protocols http://ow.ly/xkGjg

Robert F. Luo, Rajiv L. Gaur and Niaz Banaei

Clinical Microbiology Laboratory, Stanford University School of Medicine, Palo Alto, CA, USA.

Correspondence: Niaz Banaei, Stanford University Clinical Microbiology Laboratory, 3375 Hillview Avenue, Room 1602, Palo Alto, CA 94304, USA. E-mail: niazbanaei@stanford.edu

Received: April 072014 | Accepted after revision: May 212004

Conflict of interest: None declared.

\section{References}

1 Denkinger CM, Schumacher SG, Boehme CC, et al. Xpert MTB/RIF assay for the diagnosis of extrapulmonary tuberculosis: a systematic review and meta-analysis. Eur Respir J 2014; 44: 435-446.

2 World Health Organization. Automated real-time nucleic acid amplification technology for rapid and simultaneous detection of tuberculosis and rifampicin resistance: Xpert MTB/RIF assay for the for the diagnosis of pulmonary and extrapulmonary TB in adults and children. Geneva, WHO, 2013.

3 Weyer K, Mirzayev F, Migliori GB, et al. Rapid molecular TB diagnosis: evidence, policy making and global implementation of Xpert MTB/RIF. Eur Respir J 2013; 42: 252-271.

4 Patel VB, Theron G, Lenders L, et al. Diagnostic accuracy of quantitative PCR (Xpert MTB/RIF) for tuberculous meningitis in a high burden setting: a prospective study. PLoS One Med 2013; 10: e1001536.

5 World Health Organization. The use of the Xpert MTB/RIF assay for the detection of pulmonary and extrapulmonary tuberculosis and rifampicin resistance in adults and children. Expert group meeting report, 2013. www.stoptb.org/wg/gli/assets/documents/Xpert\%20Meeting\%20Report\%2024102013\%20\%20Pre\%20publication\% 20FINAL.pdf Date last updated: October 2013. Date last accessed: July 6, 2014.

Eur Respir J 2014; 44: 1094-1095 | DOI: 10.1183/09031936.00066214 | Copyright (c)ERS 2014

From the authors:

We appreciate the response from R.F. Luo and co-workers on our meta-analysis on the accuracy of Xpert MTB/RIF for extrapulmonary tuberculosis [1], as well as the data they present on various approaches to cerebrospinal fluid (CSF) processing for Xpert MTB/RIF testing.

In a recent guidance document on Xpert, the World Health Organization (WHO) recommend that Xpert should be used as a first-line test over conventional microscopy and culture in patients with suspected tuberculous meningitis $[2,3]$. This recommendation was based on a systematic review of the evidence and expert consensus [4]. However, our systematic review noted the highly variable sample processing methods used across and within studies, and was unable to identify the best approach for sample processing. The latter is largely due to the lack of recommendations from both the manufacturer and WHO on how to process nonrespiratory samples.

WHO has recognised the need for such guidance and has published an Xpert MTB/RIF implementation manual with recommendations on the technical and operational "how to", which includes standard operating procedures for processing of CSF, lymph node samples and other tissues [5]. While this is a good step forward, the recommendations are based on expert opinion and limited experimental data on the optimisation of sample preparation comparing different protocols on the same clinical samples or spiked samples in a controlled laboratory setting.

The data by R.F. Luo and co-workers addresses this knowledge gap. In a controlled laboratory environment comparing different protocols on CSF, they were not able to reproduce the finding in our systematic review of an increased sensitivity of Xpert on CSF with a centrifugation step prior to inoculation with the sample 